\title{
TRANSITION LAYER SOLUTIONS OF A HIGHER ORDER EQUATION IN AN INFINITE TUBE
}

\author{
Peter W. Bates* \\ Department of Mathematics \\ Brigham Young University \\ Provo, Utah 84602
}

\author{
Xiaofeng Ren ${ }^{\dagger}$ \\ Department of Mathematics \\ Brigham Young University \\ Provo, Utah 84602
}

\section{Introduction}

We are concerned with the higher order variational problem

$$
I=\inf _{u \in \mathcal{A}} \int_{\Omega}\left[\sum_{i, j=1}^{N} \sum_{\substack{|\alpha|=2 i-1 \\|\beta|=2 j-1}} A_{\alpha \beta} D^{\alpha} u D^{\beta} u+W(u)\right] d x .
$$

The integral in the right side of (1.1) is denoted by $E(u)$, the energy of $u$, in this paper. This problem has its origin in phase transition theory. The function $u$ is the phase field of a binary material, and $E(u)$ represents the total energy of the material, assuming the temperature is constant. This energy has two parts: the interaction energy and the bulk energy.

$$
\int_{\Omega} \sum_{i, j=1}^{N} \sum_{\substack{|\alpha|=2 i-1 \\|\beta|=2 j-1}} A_{\alpha \beta} D^{\alpha} u D^{\beta} u d u
$$

represents the interaction energy of the phase field and

$$
\int_{\Omega} W(u) d x
$$

represents the bulk energy of the phase field. Here we use multi-index notation: $\alpha=\left(\alpha_{1}, \alpha_{2}, \ldots, \alpha_{d}\right), D^{\alpha}=\partial_{x_{1}}^{\alpha_{1}} \partial_{x_{2}}^{\alpha_{2}} \ldots \partial_{x_{d}}^{\alpha_{d}}$. We assume the matrix $\left[A_{\alpha \beta}\right]$ is symmetric and positive definite, which, as we show below, arises naturally in the derivation of (1.1).

\footnotetext{
* Supported in part by NSF grant DMS-9305044.

${ }^{\dagger}$ Current address: IMA, University of Minnesota, Minneapolis, MN 55455.
} 
The bulk energy density function $W$ is assumed to be a balanced double well potential function, e.g. $W(u)=\left(u^{2}-1\right)^{2}$. $W$ has two global minima scaled to be at -1 and 1 . We assume $W$ is continuous on $R$ and $C^{2}$ near -1 and 1 , $W \geq 0,-1$ and 1 are the only global minima of $W$, and $W(-1)=W(1)=0$, $W^{\prime \prime}(-1)>0$ and $W^{\prime \prime}(1)>0$. Concerning the behavior of $W$ near infinity, we assume that

$$
\liminf _{y \rightarrow-\infty} W(y)>0 \text { and } \liminf _{y \rightarrow \infty} W(y)>0 .
$$

We also need a key assumption

$$
N>\frac{d+2}{4}
$$

on the order of the problem.

In this paper we study the variational problem in a $d$-dimensional tube of infinite length, so we will take the physical domain $\Omega$ to be an infinite tube. Because we are interested in the transitions of the phase field, we assume that $\mathcal{A}$ is a class of functions that take values -1 at one end of the tube and 1 at the other end. The precise definitions of $\Omega$ and $\mathcal{A}$ will be given later. Let us first recall how (1.1) is derived from a microscopic viewpoint.

In the theory of phase transitions for binary materials from a microscopic viewpoint, one considers a state to be a distribution $u$ over a lattice so that $u(r)$ is the probability that a solute atom occurs at site $r$. The Helmholtz free energy of a state is given by

$$
F=H-T S
$$

where $H(u)$ is the internal interaction energy, $T$ is the absolute temperature and $S(u)$ is the entropy. Following Khachaturyan (see [8] and [9] for instance), the internal energy takes the form

$$
H(u)=-\frac{1}{2} \sum_{r, r^{\prime}} J\left(r-r^{\prime}\right) u(r) u\left(r^{\prime}\right)
$$

where the sum is over the lattice sites and $J\left(r-r^{\prime}\right)=J\left(r^{\prime}-r\right)>0$ gives the pairwise interaction energies.

The total entropy takes the form of a sum over the lattice of

$$
-K[u(r) \log u(r)+(1-u(r)) \log (1-u(r))],
$$

where $K$ is a positive constant, which has minima at 0 and 1 and which naturally penalizes values of $u$ outside the interval $[0,1]$.

In the analysis to follow we will consider a rearrangement of (1.1) changing $H$ to

$$
\frac{1}{4} \sum_{r, r^{\prime}} J\left(r-r^{\prime}\right)\left(u(r)-u\left(r^{\prime}\right)\right)^{2}
$$

so that $F$ is written

$$
\begin{gathered}
\frac{1}{4} \sum_{r, r^{\prime}} J\left(r-r^{\prime}\right)\left(u(r)-u\left(r^{\prime}\right)\right)^{2} \\
+\sum_{r}\left(T K[u(r) \log u(r)+(1-u(r)) \log (1-u(r))]-c u^{2}(r)\right),
\end{gathered}
$$


where $c=(1 / 4) \sum_{r} J(r)$, which we assume is finite. Note that for $T K$ small the second term is the sum over the lattice of a double well function of $u$. We will write this term as

$$
\frac{1}{4} \sum_{r} W(u(r))
$$

and assume that $W$ is everywhere defined. For computational ease later, we re-scale $u$ so that the minima of the double well function occur at $u=-1,1$. Taking the continuum limit gives

$$
F(u)=\frac{1}{4}\left[\iint J(x-y)(u(x)-u(y))^{2} d x d y+\int W(u(x)) d x\right] .
$$

We assume $J$ is non-negative, symmetric about 0 , and $\int J(x) d x=1$.

As was done in [2], we change variables in the first integral using $\eta=(x-$ $y) / 2, \xi=(x+y) / 2$, then expand $u(x)=u(\xi+\eta)$ and $u(y)=u(\xi-\eta)$ about $\xi$ to get

$$
2^{d} \iint J(2 \eta)\left(\sum_{k=1}^{\infty} \sum_{|\alpha|=2 k-1} \frac{1}{\alpha !} D^{\alpha} u(\xi) \eta^{\alpha}\right)^{2} d \xi d \eta
$$

where both integrals are over $R^{d}, \alpha=\left(\alpha_{1}, \alpha_{2}, \ldots, \alpha_{d}\right),|\alpha|=\alpha_{1}+\alpha_{2}+\ldots+\alpha_{d}$, $D^{\alpha}=\partial_{x_{1}}^{\alpha_{1}} \partial_{x_{2}}^{\alpha_{2}} \ldots \partial_{x_{d}}^{\alpha_{d}}, \alpha !=\alpha_{1} ! \alpha_{2} ! \ldots \alpha_{d} !$, and $\eta^{\alpha}=\eta_{1}^{\alpha_{1}} \eta_{2}^{\alpha_{2}} \ldots \eta_{d}^{\alpha_{d}}$.

We truncate the infinite sum at $k=N$ and integrate with respect to $\eta$. This "truncated" free energy is given by (1.1) where

$$
A_{\alpha \beta}=2^{d} \int \frac{J(2 \eta) \eta^{\alpha+\beta}}{\alpha ! \beta !} d \eta
$$

Note that $\left[A_{\alpha \beta}\right]$ is positive definite since

$$
\sum_{i, j=1}^{N} \sum_{\substack{|\alpha|=2 i-1 \\|\beta|=2 j-1}} A_{\alpha \beta} \xi_{\alpha} \xi_{\beta}=2^{d} \int J(2 \eta)\left(\sum_{i=1}^{N} \sum_{|\alpha|=2 i-1} \frac{\eta^{\alpha} \xi_{\alpha}}{\alpha !}\right)^{2} d \eta \geq 0
$$

and the equality holds only if $\xi_{\alpha}=0$ for all $\alpha$.

The Euler-Lagrange equation of (1.1) is

$$
\left\{\begin{array}{l}
\sum_{\substack{i, j=1 \\
|\alpha|=2 i-1 \\
|\beta|=2 j-1}}^{N} 2 A_{\alpha \beta} D^{\alpha+\beta} u-W^{\prime}(u)=0 \text { in } \Omega \\
B_{j}(u)=0 \text { on } \partial \Omega, j=1,2, \ldots, 2 N-1
\end{array}\right.
$$

where the $B_{j}$ 's are the natural boundary conditions. The expressions of the $B_{j}$ 's are complicated. In the case $d=2, N=2$ and the material is isotropic, i.e., $J(x)=J(|x|)$, we have

$$
B_{1}(u)=\frac{b}{16} \frac{\partial u}{\partial \nu}+\frac{c}{3} \nu_{1}^{2} \frac{\partial u_{y y}}{\partial \nu}+\frac{c}{3} \nu_{2}^{2} \frac{\partial u_{x x}}{\partial \nu}
$$




$$
\begin{gathered}
+\nu_{1}^{2}\left(\frac{5 c}{9} \nu_{1} u_{x x x}+c \nu_{2} u_{x x y}\right)+\nu_{2}^{2}\left(\frac{5 c}{9} \nu_{2} u_{y y y}+c \nu_{1} u_{x y y}\right) \\
B_{2}(u)=\frac{\partial}{\partial \tau}\left[-\frac{10 c}{9} \nu_{1}^{2} \nu_{2} u_{x x x}-2 c \nu_{1} \nu_{2}^{2} u_{x x y}+\frac{10 c}{9} \nu_{1}^{2} \nu_{2} u_{y y y}\right. \\
\left.+2 c \nu_{1}^{2} \nu_{2} u_{x y y}+\frac{2 c}{3} \nu_{1} \nu_{2} \frac{\partial u_{x x}}{\partial \nu}-\frac{2 c}{3} \nu_{1} \nu_{2} \frac{\partial u_{y y}}{\partial \nu}\right] \\
-\nu_{1}^{2}\left(\frac{5 c}{9} u_{x x x}+c u_{x x y}\right)-\nu_{2}^{2}\left(\frac{5 c}{9} u_{y y y}+c u_{x y y}\right)-\frac{c}{3} \nu_{2}^{2} \Delta u_{x x}-\frac{c}{3} \nu_{1}^{2} \Delta u_{y y} \nu_{1}^{2}-\frac{b}{16} \Delta u \\
B_{3}(u)=\frac{\partial^{2}}{\partial \tau^{2}}\left[\nu_{2}^{2}\left(\frac{5 c}{9} \nu_{1} u_{x x x}+c \nu_{2} u_{x x x y} \nu_{2}\right)+\nu_{1}^{2}\left(\frac{5 c}{9} \nu_{2} u_{y y y}+c \nu_{1} u_{x y y}\right)\right. \\
\left.+\nu_{1}^{2}\left(\frac{c}{3} \frac{\partial u_{x x}}{\partial \nu}\right)+\nu_{2}^{2}\left(\frac{c}{3} \frac{\partial u_{y y}}{\partial \nu}\right)+\frac{b}{16} \frac{\partial u}{\partial \nu}\right] \\
+\frac{\partial}{\partial \tau}\left[\nu_{1} \nu_{2}\left(\frac{5 c}{9} u_{x x x}+c u_{x x y}\right)-\nu_{1} \nu_{2}\left(\frac{5 c}{9} u_{y y y}+c u_{x y y}\right)+\frac{c}{3} \nu_{1} \nu_{2}\left(-\Delta u_{x x}+\Delta u_{y y}\right)\right] \\
+\nu_{1}\left(\frac{5 c}{9} u_{x x x x x}+c u_{x x x y y}\right)+\nu_{2}\left(\frac{5 c}{9} u_{y y y y y}+c u_{x x y y y}\right) \\
+\frac{c}{3} \Delta u_{x x y} \nu_{2}+\frac{c}{3} \Delta u_{x y y} \nu_{1}+\frac{b}{8} \frac{\partial(\Delta u)}{\nu}+\frac{a}{2} \frac{\partial u}{\partial \nu}
\end{gathered}
$$

where $a, b, c$ are three constants, $\nu=\left(\nu_{1}, \nu_{2}\right)$ is the normal vector to $\partial \Omega$ pointing outward, and $\tau=\left(\nu_{2},-\nu_{1}\right)$ is the unit tangential vector that makes $(\tau, \nu)$ positively oriented.

This paper is devoted to the existence of the energy minimizers of (1.1), or the solutions of (1.5). The simplest case $\Omega=R$ and $N=1$ gives the equation

$$
\left\{\begin{array}{l}
u^{\prime \prime}-W^{\prime}(u)=0 \text { in } R \\
\lim _{x \rightarrow-\infty} u(x)=-1, \lim _{x \rightarrow \infty} u(x)=1 .
\end{array}\right.
$$

It was shown by Fife and McLeod in [6] that this equation has a unique solution up to translation and the manifold of all the translates is globally stable. Indeed they considered the more general case where $W$ is not necessarily balanced and they considered traveling wave solutions. Note that if $W$ is balanced, the traveling wave solutions become standing waves (the speed is 0 ).

Gardner and Jones studied the case where $\Omega=R$ and $N=2$ in [7]. They also considered traveling wave solutions when $W$ is not necessarily balanced. Bates, Fife, Gardner and Jones [3] generalized this case to arbitrary N. In both papers the higher order equations were treated as the perturbations of the second order equation and the existence and stability of traveling wave solutions was obtained if the coefficients of the higher order derivatives were "small".

In [5], the authors studied the case where $W$ is balanced, $\Omega=R$, and $N$ is arbitrary, but without the smallness condition on the higher order derivatives. We proved the existence of energy minimizing solutions having limits of -1 , 1 at $-\infty, \infty$, by using the concentration-compactness principle to capture an energy minimizer from any minimizing sequence. 
In this paper we consider a domain $\Omega$ which is a tube of infinite length in $R^{d}$. We will consider two kinds of tubes. In section 3, we study tubes which are periodic in one direction. The discrete translational symmetry allows us to prove the existence of the minimizers by taking appropriate translates of terms in a minimizing sequence. Section 4 contains the proof of a lemma used in section 3 . Then in section 5 , we consider tubes whose tails are "half-periodic", and we give a sufficient condition on the geometry of the tube for the existence of energy minimizers. We also give various examples where such a condition is satisfied.

\section{Concentration functions of $\left\{W\left(u_{n}\right)\right\}$}

$\Omega$ is assumed to be a sufficiently smooth open connected set in $R^{d}$. We will sometimes treat $\Omega$ as a metric space equipped with a distance function $\rho$ : $\Omega \times \Omega \rightarrow R$ defined by

$$
\rho(x, y)=\inf _{\gamma \in \Gamma} \int_{0}^{1}\left|\gamma^{\prime}(t)\right| d t
$$

where $\Gamma$ is the class of all smooth paths $\gamma:[0,1] \rightarrow \Omega$ with $\gamma(0)=x$ and $\gamma(1)=y$. We define

$$
B_{\Omega}(x, r)=\{y \in \Omega: \rho(y, x)<r\}
$$

to be the ball centered at $x$ with radius $r$ in the metric space $(\Omega, \rho)$. If we denote the usual ball in $R^{d}$ centered at $x$ with radius $r$ by $B(x, r)$, in general $B_{\Omega}(x, r) \neq B(x, r) \cap \Omega$.

To say $\Omega$ is a tube, we assume that: (1) there is a large ball $B$ in $R^{d}$ such that $\Omega \backslash \bar{B}$ consists of two unbounded connected open sets $\Omega_{1}$ and $\Omega_{2} ;(2)$ there exist $P_{i}$ and $Q_{i}$ such that $P_{i} \subset \Omega_{i} \subset Q_{i}$ where $P_{i}$ and $Q_{i}$ are diffeomorphic to the half infinite cylinder $[0, \infty) \times D \subset R^{d}$, with $D$ being the unit ball in $R^{d-1}$; (3) the diffeomorphisms have bounded gradients. This means that neither end of the tube will shrink to a point at infinity. We assume throughout this paper that $\Omega$ is a tube.

To prescribe the condition of the phase fields at the two ends of the tube $\Omega$, we denote the two ends of $\Omega$ by $E_{1}$ and $E_{2}$. We use $x \rightarrow E_{i}$ to denote $x$ approaching $E_{i}$ uniformly.

To define the function class $\mathcal{A}$ in (1.1), we first state the following lemma.

Lemma 2.1 If $u$ is a function in $L_{\text {loc }}^{1}(\Omega)$ with $W(u) \in L^{1}(\Omega), D^{\alpha} u \in L^{2}$ for all $\alpha,|\alpha|=2 i-1, i=1,2, \ldots, N$, then $\lim _{x \rightarrow E_{1}} u(x)$ and $\lim _{x \rightarrow E_{2}} u(x)$ exist and equal -1 or 1 .

Proof. Recall an interpolation inequality for intermediate derivatives (see theorem 4.14 [1]): Let $\Omega \subset R^{d}$ have the uniform cone property, and let $\epsilon_{0}$ be a finite 
positive number. Then there exists a constant $K=K\left(\epsilon_{0}, m, p, \Omega\right)$ such that for any $\epsilon, 0<\epsilon \leq \epsilon_{0}$, any integer $j, 0 \leq j \leq m-1$, and any $v \in W^{m, p}(\Omega)$

$$
|v|_{j, p} \leq K \epsilon|v|_{m, p}+K \epsilon^{-j}(m-j)|v|_{0, p}
$$

where

$$
|v|_{j, p}=\left\{\sum_{|\alpha|=j} \int_{\Omega}\left|D^{\alpha} v\right|^{p} d x\right\}^{1 / p} .
$$

With the help of this inequality, a standard smoothing argument implies that for every $u$ in lemma $2.1, D^{\alpha} u \in L^{2}(\Omega),|\alpha|=1,2, \ldots, 2 N-1$. We now apply the Sobolev embedding theorem (see theorem 5.4 [1]) to conclude that there is $\sigma, 0<\sigma<1$, such that

$$
\sup _{x, y \in \Omega} \frac{|u(x)-u(y)|}{|x-y|^{\sigma}}<\infty
$$

Here we used our key assumption (1.4). Therefore we have a uniform bound on the modulus of continuity of $u$. Since $\int_{\Omega} W(u)<\infty$, we deduce that $\lim _{x \rightarrow E_{1}} u(x), \lim _{x \rightarrow E_{2}} u(x)$ exist and equal -1 or 1 .

We now define

$$
\mathcal{A}^{*}=\left\{u \in L_{l o c}^{1}(\Omega): D^{\alpha} u \in L^{2}(\Omega),|\alpha|=2 i-1, i=1, \ldots, N, \int_{\Omega} W(u)<\infty\right\} .
$$

In view of lemma 2.1 , we can decompose $\mathcal{A}^{*}$ in to four mutually disjoint classes, $\mathcal{A}, \mathcal{A}^{\prime}, \mathcal{A}^{\prime \prime}$ and $\mathcal{A}^{\prime \prime \prime}$, where

$$
\left\{\begin{array}{l}
\mathcal{A}=\left\{u \in \mathcal{A}: \lim _{x \rightarrow E_{1}} u(x)=-1 \lim _{x \rightarrow E_{2}} u(x)=1\right\}, \\
\mathcal{A}^{\prime}=\left\{u \in \mathcal{A}: \lim _{x \rightarrow E_{1}} u(x)=1 \lim _{x \rightarrow E_{2}} u(x)=-1\right\}, \\
\mathcal{A}^{\prime \prime}=\left\{u \in \mathcal{A}: \lim _{x \rightarrow E_{1}} u(x)=1 \lim _{x \rightarrow E_{2}} u(x)=1\right\}, \\
\mathcal{A}^{\prime \prime \prime}=\left\{u \in \mathcal{A}: \lim _{x \rightarrow E_{1}} u(x)=-1 \lim _{x \rightarrow E_{2}} u(x)=-1\right\} .
\end{array}\right.
$$

We are only interested in the first class, $\mathcal{A}$.

We now give a necessary condition for a phase transition to occur.

Lemma 2.2 For a given energy minimizing sequence $\left\{u_{n}\right\}$ and every $\epsilon_{1}>0$, $L>0$ there exists $\delta>0$ so that for every $n$ and $B_{\Omega}(y, r) \subset \Omega$, with $L<r \leq \infty$,

$$
\int_{B_{\Omega}(y, r)} W\left(u_{n}\right) d x<\delta
$$

implies that either

$$
u_{n}(x) \in\left(-1-\epsilon_{1},-1+\epsilon_{1}\right)
$$

for all $x \in B_{\Omega}(y, r)$ or

$$
u_{n}(x) \in\left(1-\epsilon_{1}, 1+\epsilon_{1}\right)
$$

for all $x \in B_{\Omega}(y, r)$. 
Proof. Suppose the contrary. There exist $\epsilon_{1}, L, \delta_{k}, \delta_{k} \rightarrow \infty$ as $k \rightarrow \infty, u_{n_{k}}$ from the minimizing sequence, $B_{\Omega}\left(y_{k}, r_{k}\right), L \leq r_{k} \leq \infty$, and $x_{k}, x_{k} \in B_{\Omega}\left(y_{k}, r_{k}\right)$, such that

$$
\int_{B_{\Omega}\left(y_{k}, r_{k}\right)} W\left(u_{n_{k}}\right) d x \leq \delta_{k} \rightarrow 0 \text { as } k \rightarrow \infty
$$

and $u_{n_{k}}\left(x_{k}\right) \notin\left(-1-\epsilon_{1},-1+\epsilon_{1}\right) \cup\left(1-\epsilon_{1}, 1+\epsilon_{1}\right)$. Because we have a bound independent of $k$ on the moduli of continuity of the $u_{n_{k}}$ 's, (2.4), we can find $\eta$ independent of $k$ such that for all $x \in B_{\Omega}\left(x_{k}, \eta\right),\left|u_{n_{k}}(x)-1\right| \geq \epsilon_{1} / 2$ and $\left|u_{n_{k}}(x)+1\right| \geq \epsilon_{1} / 2$, so we deduce

$$
\begin{gathered}
\int_{B_{\Omega}\left(y_{k}, r_{k}\right)} W\left(u_{n_{k}}\right) d x \geq \int_{B_{\Omega}\left(x_{k}, \eta\right) \cap B_{\Omega}\left(y_{k}, r_{k}\right)} W\left(u_{n_{k}}\right) d x \\
\geq C \eta^{d} \min _{x \in B_{\Omega}\left(x_{k}, \eta\right) \cap B_{\Omega}\left(y_{k}, r_{k}\right)} W\left(u_{n_{k}}(x)\right) \\
\geq C \eta^{d} \min _{y \in R \backslash\left(-1-\epsilon_{1} / 2,-1+\epsilon_{1} / 2\right) \cup\left(1-\epsilon_{1} / 2,1+\epsilon_{1} / 2\right)} W(y) \geq C^{\prime}>0
\end{gathered}
$$

where $C$ depends on $d$ only. This contradicts (2.6).

Our next lemma asserts that $\left\{\int_{\Omega} W\left(u_{n}\right)\right\}$ does not vanish.

Lemma 2.3 For any energy minimizing sequence $\left\{u_{n}\right\} \subset \mathcal{A}$ there exists $\Lambda \in$ $(0, \infty)$ such that for all $n$

$$
\int_{\Omega} W\left(u_{n}\right) d x \geq \Lambda
$$

Proof. Since $I<\infty, \lim \sup _{n \rightarrow \infty} \int_{\Omega} W\left(u_{n}\right) d x<\infty$. For every $u \in \mathcal{A}$ there is $x_{n} \in \Omega$ such that $u_{n}\left(x_{n}\right)=0$. (2.4) yields a bound independent of $n$ on the moduli of continuity of the $u_{n}$ 's. Hence there exist $\epsilon_{1}>0, \delta>0$, both independent of $n$, such that $u_{n}(x) \in\left(-\epsilon_{1}, \epsilon_{1}\right)$ for $x \in B_{\Omega}\left(x_{n}, \delta\right)$. This implies $\Lambda>0$.

We now apply the concentration-compactness principle to $\left\{W\left(u_{n}\right)\right\}$. In lemma 2.3 , we may take

$$
\Lambda=\liminf _{n \rightarrow \infty} \int_{\Omega} W\left(u_{n}\right) d x
$$

and then take $\left\{u_{n}\right\}$ to be a subsequence along which this is achieved. We treat $\left\{W\left(u_{n}\right)\right\}$ as a sequence of non-negative functions in $L^{1}(\Omega)$. Define the concentration functions

$$
Q_{n}(t)=\sup _{y \in \Omega} \int_{B_{\Omega}(y, t)} W\left(u_{n}(x)\right) d x
$$

for $t \geq 0 . \quad\left\{Q_{n}\right\}$ is a sequence of non-negative, non-decreasing, uniformly bounded functions on $[0, \infty)$ and

$$
\lim _{n \rightarrow \infty} \lim _{t \rightarrow \infty} Q_{n}(t)=\Lambda .
$$


By a classical result about monotone functions, there exist a subsequence of $u_{n}$, again denoted by $u_{n}$, and a non-negative, non-decreasing function $Q(t)$ such that

$$
\lim _{n \rightarrow \infty} Q_{n}(t)=Q(t)
$$

for all $t \geq 0$. Let

$$
\lambda=\lim _{t \rightarrow \infty} Q(t) .
$$

Then $\lambda \in[0, \Lambda]$. Furthermore by (2.9) and (2.10) we may make the following three statements:

I: If $\lambda=\Lambda$, there exist a subsequence of $u_{n}$, again denoted by $u_{n}$, and $y_{n} \in \Omega$ such that for every $\epsilon>0$ there exist $r>0$ and a positive integer $n_{1}$ so that

$$
\int_{B_{\Omega}\left(y_{n}, r\right)} W\left(u_{n}(x)\right) d x \geq \Lambda-\epsilon
$$

for $n \geq n_{1}$.

II: If $\lambda=0$, then for all $r>0$,

$$
\lim _{n \rightarrow \infty} \sup _{y \in \Omega} \int_{B_{\Omega}(y, r)} W\left(u_{n}(x)\right) d x=0 .
$$

III: If $\lambda \in(0, \Lambda)$, then there exists a subsequence of $\left\{u_{n}\right\}$ again denoted by $\left\{u_{n}\right\}$ such that for every $\epsilon>0$ there exist arbitrarily large $r>0$, a sequence $r_{n} \rightarrow \infty$ as $n \rightarrow \infty$, and a positive integer $n_{1}$ such that for $n \geq n_{1}$

$$
\lambda-\epsilon<Q_{n}(r), Q_{n}\left(r_{n}\right)<\lambda+\epsilon .
$$

These three statements can be found in the proof of lemma I.1 [10].

\section{An existence theorem for periodic $\Omega$}

Throughout section 3, we assume the tube $\Omega$ is periodic in one direction. This translation invariance enables us to construct an energy minimizer.

We suppose there is $T \in R^{d}$ such that $\Omega+T=\Omega$ where $\Omega+T=\{x+T$ : $x \in \Omega\}$ is the translation of $\Omega$ by $T$. To say $\Omega$ is a tube, we assume the cross sections of $\Omega$ perpendicular to $T$ are bounded (see figure 1). Our main result in this section is the following theorem.

Theorem 3.1 If the tube $\Omega$ is periodic, every energy minimizing sequence of (1.1) gives rise to an energy minimizer in $\mathcal{A}$. Indeed if $\left\{u_{n}\right\}$ is an energy minimizing sequence, there exist a subsequence of $\left\{u_{n}\right\}$, again denoted by $\left\{u_{n}\right\}$, and a sequence of integers $\left\{i_{n}\right\}$ such that $\left\{u_{n}\left(\cdot+i_{n} T\right)\right\}$ converges in a suitable sense to an energy minimizer. 


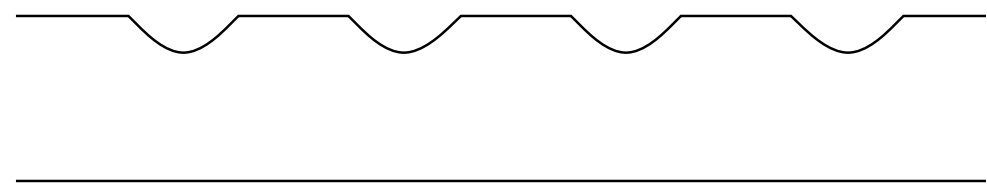

Figure 1: An example of periodic $\Omega$.

We will prove theorem 3.1 by showing that only the case $\lambda=\Lambda$ in statement I in section 2 can occur when $\left\{u_{n}\right\}$ is an energy minimizing sequence.

Lemma 3.2 The case $\lambda=0$ can not occur.

Proof. As in the proof of lemma 2.3, we can find $x_{n}$, and $\delta$ independent of $n$, such that $\int_{B_{\Omega}\left(x_{n}, \delta\right)} W\left(u_{n}\right) d x \geq C$ for some $C$ independent of $n$.

Lemma 3.3 The case $\lambda \in(0, \Lambda)$ can not occur.

The proof of this lemma is postponed to section 4 .

Lemma 3.4 The case $\lambda=\Lambda$ implies that there is $u \in \mathcal{A}$ such that

$$
E(u)=\inf _{v \in \mathcal{A}} E(v) .
$$

Proof. Statement I in section 2 implies that for every $\epsilon>0$ there exist $\left\{y_{n}\right\}$, $r>0$ and a positive integer $n_{1}$ such that for $n \geq n_{1}$

$$
\int_{B_{\Omega}\left(y_{n}, r\right)} W\left(u_{n}\right) \geq \Lambda-\epsilon
$$

Using the periodicity of $\Omega$ and replacing $r$ by $r+D_{T}$, we can translate the $u_{n}$ 's by $i_{n} T$ where the $i_{n}$ 's are some integers, $T$ is the period of the tube and $D_{T}$ is the diameter of a piece of the tube of "length" $T$, and therefore take $y_{n}$ to be a point $y$ independent of $n$. By further enlarging $r$, we may assume that $\Omega$ is divided into mutually disjoint open sets $\Omega_{1}, \Omega_{2}$ and $B_{\Omega}(y, r)$ with

$$
\bar{\Omega}=\bar{\Omega}_{1} \cup \bar{\Omega}_{2} \cup \bar{B}_{\Omega}(y, r) .
$$

Assume $E_{1}$ is associated to $\Omega_{1}$ and $E_{2}$ is associated to $\Omega_{2}$. For large $n$

$$
\int_{\Omega_{1}} W\left(u_{n}\left(x+i_{n} T\right)\right) d x<2 \epsilon, \int_{\Omega_{2}} W\left(u_{n}^{\prime}\left(x+i_{n} T\right)\right) d x<2 \epsilon .
$$

Lemma 2.2 then implies that the $u_{n}\left(\cdot+i_{n} T\right)$ 's stay close to -1 in $\Omega_{1}$ and close to 1 in $\Omega_{2}$ uniformly in $n$. We pass to the limit along a subsequence and obtain $u \in \mathcal{A}$ such that

$$
u_{n}\left(\cdot+i_{n} T\right) \rightarrow u \text { in } C(K \cap \Omega) \text { for every compact } K \in R^{d},
$$


$D^{\alpha} u_{n}\left(\cdot+i_{n} T\right) \rightarrow u$ weakly in $L^{2}(\Omega),|\alpha|=2 i-1, i=1,2, \ldots, N$.

The positivity of $\left[A_{\alpha \beta}\right]$ and Fatou's lemma imply

$$
E(u) \leq \lim _{n \rightarrow \infty} E\left(u_{n}\right)=I,
$$

so $u$ is an energy minimizer.

Once we have an energy minimizer $u$, we know that $u$ solves the corresponding Euler-Lagrange equation and the proof of theorem 3.1 is complete.

Corollary 3.5 The convergence in (3.1) and (3.2) is indeed

$$
\begin{gathered}
u_{n}\left(\cdot+i_{n} T\right) \rightarrow u \text { in } C(\bar{\Omega}), \\
D^{\alpha} u_{n}\left(\cdot+i_{n} T\right) \rightarrow D^{\alpha} u \text { strongly in } L^{2}(\Omega),|\alpha|=2 i-1, i=1,2, \ldots, N .
\end{gathered}
$$

Proof. The uniform estimate of the $u_{n}\left(\cdot+i_{n} T\right)$ 's in $\Omega_{1}$ and $\Omega_{2}$ gives the convergence in $C(\bar{\Omega})$. To see the strong convergence in $L^{2}(\Omega)$, one notes that

$$
\begin{gathered}
\lim _{n \rightarrow \infty} \int_{\Omega} \sum_{i, j=1}^{N} \sum_{\substack{|\alpha|=2 i-1 \\
|\beta|=2 j-1}} A_{\alpha \beta} D^{\alpha} u_{n} D^{\beta} u_{n} d x \\
=\int_{\Omega} \sum_{i, j=1}^{N} \sum_{\substack{|\alpha|=2 i-1 \\
|\beta|=2 j-1}} A_{\alpha \beta} D^{\alpha} u D^{\beta} u d x .
\end{gathered}
$$

We then consider

$$
\begin{gathered}
\int_{\Omega} \sum_{i, j=1}^{N} \sum_{\substack{|\alpha|=2 i-1 \\
|\beta|=2 j-1}} A_{\alpha \beta} D^{\alpha}\left(u_{n}-u\right) D^{\beta}\left(u_{n}-u\right) d x \\
=\int_{\Omega} \sum_{i, j=1}^{N} \sum_{\substack{|\alpha|=2 i-1 \\
|\beta|=2 j-1}} A_{\alpha \beta} D^{\alpha} u_{n} D^{\beta} u_{n} d x+\int_{\Omega} \sum_{i, j=1}^{N} \sum_{\substack{|\alpha|=2 i-1 \\
|\beta|=2 j-1}} A_{\alpha \beta} D^{\alpha} u D^{\beta} u d x \\
-\int_{\Omega} \sum_{i, j=1}^{N} \sum_{\substack{|\alpha|=2 i-1 \\
|\beta|=2 j-1}} A_{\alpha \beta} D^{\alpha} u_{n} D^{\beta} u d x-\int_{\Omega} \sum_{i, j=1}^{N} \sum_{\substack{|\alpha|=2 i-1 \\
|\beta|=2 j-1}} A_{\alpha \beta} D^{\alpha} u D^{\beta} u_{n} d x .
\end{gathered}
$$

(3.2) implies

$$
\begin{aligned}
& \lim _{n \rightarrow \infty} \int_{\Omega} \sum_{i, j=1}^{N} \sum_{\substack{\alpha|=2 i-1\\
| \beta \mid=2 j-1}} A_{\alpha \beta} D^{\alpha} u_{n} D^{\beta} u d x=\int_{\Omega} \sum_{i, j=1}^{N} \sum_{\substack{\alpha|=2 i-1\\
| \beta \mid=2 j-1}} A_{\alpha \beta} D^{\alpha} u D^{\beta} u d x, \\
& \lim _{n \rightarrow \infty} \int_{\Omega} \sum_{i, j=1}^{N} \sum_{\substack{|\alpha|=2 i-1 \\
|\beta|=2 j-1}} A_{\alpha \beta} D^{\alpha} u D^{\beta} u_{n} d x=\int_{\Omega} \sum_{i, j=1}^{N} \sum_{\substack{\alpha|=2 i-1\\
| \beta \mid=2 j-1}} A_{\alpha \beta} D^{\alpha} u D^{\beta} u d x .
\end{aligned}
$$


Therefore with the help of (3.3) we deduce

$$
\lim _{n \rightarrow \infty} \int_{\Omega} \sum_{i, j=1}^{N} \sum_{\substack{\alpha|=2 i-1\\| \beta \mid=2 j-1}} A_{\alpha \beta} D^{\alpha}\left(u_{n}-u\right) D^{\beta}\left(u_{n}-u\right) d x=0 .
$$

The positivity of $\left[A_{\alpha \beta}\right]$ implies that there exists $c>0$ such that

$$
\sum_{i, j=1}^{N} \sum_{\substack{\alpha|=2 i-1\\| \beta \mid=2 j-1}} A_{\alpha \beta} D^{\alpha}\left(u_{n}-u\right) D^{\beta}\left(u_{n}-u\right) \geq c \sum_{i=1}^{N} \sum_{|\alpha|=2 i-1}\left|D^{\alpha}\left(u_{n}-u\right)\right|^{2}
$$

With the help of (3.4) we obtain

$$
\lim _{n \rightarrow \infty} \int_{\Omega} \sum_{i=1}^{N} \sum_{|\alpha|=2 i-1}\left|D^{2 i-1}\left(u_{n}-u\right)\right|^{2}=0
$$

which gives the strong convergence.

\section{$4 \quad$ Proof of lemma 3.3}

Suppose $\lambda \in(0, \Lambda)$. From statement III in section 2, we have for every small $\epsilon>0$, numbers $r>0$, and $r_{n}$, with $r_{n} \rightarrow \infty$ as $n \rightarrow \infty$, satisfying

$$
\lambda-\epsilon<Q_{n}(r), Q_{n}\left(r_{n}\right)<\lambda+\epsilon
$$

for large $n$. We can find $y_{n}$ such that

$$
\begin{aligned}
& \int_{B_{\Omega}\left(y_{n}, r\right)} W\left(u_{n}\right) d x \in(\lambda-\epsilon, \lambda+\epsilon) \\
& \int_{\Omega \backslash B_{\Omega}\left(y_{n}, r_{n}\right)} W\left(u_{n}\right) d x \in(\Lambda-\lambda-2 \epsilon, \Lambda-\lambda+2 \epsilon)
\end{aligned}
$$

for large $n$. If we choose $r$ large enough we can write

$$
B_{\Omega}\left(y_{n}, r_{n}\right) \backslash \bar{B}_{\Omega}\left(y_{n}, r\right)=D_{1 n} \cup D_{2 n}
$$

where $D_{1 n}$ and $D_{2 n}$ are two connected open sets. We also denote

$$
\Omega \backslash \bar{B}_{\Omega}\left(y_{n}, r\right)=M_{1 n} \cup M_{2 n}
$$

where $M_{1 n}$ and $M_{2 n}$ are again two connected open sets. We assume $D_{1 n}\left(D_{2 n}\right.$, respectively) is contained in $M_{1 n}\left(M_{2 n}\right.$, respectively) which has tail $E_{1}\left(E_{2}\right.$, respectively). Clearly

$$
\operatorname{mes}\left(D_{1 n}\right), \operatorname{mes}\left(D_{2 n}\right) \rightarrow \infty
$$


where $\operatorname{mes}(D)$ denotes the measure of $D$. For large $n$

$$
\int_{D_{1 n}} W\left(u_{n}\right) d x<2 \epsilon, \int_{D_{2 n}} W\left(u_{n}\right) d x<2 \epsilon .
$$

If we choose $\epsilon$ small enough, we see the $u_{n}$ 's stay close to -1 or 1 in $D_{1 n}$ and $D_{2 n}$ in view of lemma 2.2. We will consider the three cases.

Case 1: Suppose, taking a subsequence if necessary, the $u_{n}$ 's stay close to -1 in $D_{2 n}$.

We will show that in this case the $u_{n}$ 's already have large energies in $M_{2 n}$ so they can not constitute an energy minimizing sequence. Without loss of generality, we assume the period $T$ of $\Omega$ is $T=\left(T^{1}, 0, \ldots, 0\right)$. Let $\xi$ be a nonnegative smooth cut-off function such that

$$
\left\{\begin{array}{l}
\xi(t)=0 \text { if } t<0 \\
\xi(t)=1 \text { if } t>1
\end{array}\right.
$$

$0 \leq \xi(t) \leq 1$, and $\left|D^{k} \xi(x)\right| \leq C_{1}$ for $k=1,2, \ldots 2 N-1$. Set

$$
v_{n}(x)=\left(u_{n}(x)+1\right) \xi\left(\frac{x^{1}-\left(y_{n}^{1}+\left(r+r_{n}\right) / 2\right)}{h}\right)-1
$$

where $x=\left(x^{1}, \ldots, x^{d}\right)$ and $y_{n}=\left(y_{n}^{1}, \ldots, y_{n}^{d}\right)$ and $h$ is a large number to be specified later. Note $v_{n} \in \mathcal{A}$. We fix $h$ and take $n$ large enough so that $h<$ $\left(r_{n}-r\right) / 2$.

We will compare $\int_{M_{2 n}} W\left(u_{n}\right) d x$ with $\int_{\Omega} W\left(v_{n}\right) d x$. Since for $x \in M_{2 n}, u_{n}$ and $v_{n}$ only differ in $D_{2 n}$, and on that set $v_{n}$ is closer to -1 than $u_{n}$ is, so we have

$$
\int_{\Omega} W\left(v_{n}\right) d x \leq \int_{M_{2 n}} W\left(u_{n}\right) d x
$$

We also compare

$$
\int_{M_{2 n}} A_{\alpha \beta} D^{\alpha} u_{n} D^{\beta} u_{n} d x \text { with } \int_{\Omega} A_{\alpha \beta} D^{\alpha} v_{n} D^{\beta} v_{n} d x .
$$

Since the two quantities agree in $M_{2 n} \backslash D_{2 n}$ and $D^{\alpha} v_{n}$ vanishes in $\Omega \backslash M_{2 n}$, we need only to compare

$$
\int_{D_{2 n}} A_{\alpha \beta} D^{\alpha} u_{n} D^{\beta} u_{n} d x \text { with } \int_{D_{2 n}} A_{\alpha \beta} D^{\alpha} v_{n} D^{\beta} v_{n} d x
$$

Consider

$$
\begin{gathered}
A_{\alpha \beta} D^{\alpha} v_{n}(x) D^{\beta} v_{n}(x)= \\
A_{\alpha \beta}\left[\sum_{\iota \leq \alpha} \frac{\alpha !}{\iota !} D^{\alpha-\iota}\left(u_{n}(x)+1\right) D^{\iota} \xi\left(\frac{x^{1}-\left(y_{n}^{1}+\left(r+r_{n}\right) / 2\right)}{h}\right)\right]
\end{gathered}
$$




$$
\begin{gathered}
\times\left[\sum_{\kappa \leq \beta} \frac{\beta !}{\kappa !} D^{\beta-\kappa}\left(u_{n}(x)+1\right) D^{\kappa} \xi\left(\frac{x^{1}-\left(y_{n}^{1}+\left(r+r_{n}\right) / 2\right)}{h}\right)\right] \\
=A_{\alpha \beta}\left[\sum_{\iota_{1} \leq \alpha_{1}} \frac{\alpha !}{\iota_{1} !} D^{\alpha-\left(\iota_{1}, 0, \ldots, 0\right)}\left(u_{n}(x)+1\right) D^{\iota_{1}} \xi\left(\frac{x^{1}-\left(y_{n}^{1}+\left(r+r_{n}\right) / 2\right)}{h}\right)\right] \\
\times\left[\sum_{\kappa_{1} \leq \beta_{1}} \frac{\beta !}{\kappa_{1} !} D^{\beta-\left(\kappa_{1}, 0 \ldots, 0\right)}\left(u_{n}(x)+1\right) D^{\kappa_{1}} \xi\left(\frac{x^{1}-\left(y_{n}^{1}+\left(r+r_{n}\right) / 2\right)}{h}\right)\right] .
\end{gathered}
$$

The first term from the product is

$$
A_{\alpha \beta} \xi^{2}\left(\frac{x^{1}-\left(y_{n}^{1}+\left(r+r_{n}\right) / 2\right)}{h}\right) D^{\alpha} u_{n} D^{\beta} u_{n}
$$

and the other terms assume the form

$$
\begin{aligned}
& \frac{A_{\alpha \beta} \alpha ! \beta !}{h^{\iota_{1}+\kappa_{1}} \iota_{1} ! \kappa_{1} !} D^{\alpha-\left(\iota_{1}, 0, \ldots, 0\right)}\left(u_{n}(x)+1\right) D^{\beta-\left(\kappa_{1}, 0 \ldots, 0\right)}\left(u_{n}(x)+1\right) \\
& \xi^{\left(\iota_{1}\right)}\left(\frac{x^{1}-\left(y_{n}^{1}+\left(r+r_{n}\right) / 2\right)}{h}\right) \xi^{\left(\kappa_{1}\right)}\left(\frac{x^{1}-\left(y_{n}^{1}+\left(r+r_{n}\right) / 2\right)}{h}\right)
\end{aligned}
$$

where at least one of $\iota_{1}$ and $\kappa_{1}$ is not equal to 0 . They can be bounded by

$$
\frac{C}{h^{\iota_{1}+\kappa_{1}}}\left|D^{\alpha-\left(\iota_{1}, 0, \ldots, 0\right)}\left(u_{n}(x)+1\right) D^{\beta-\left(\kappa_{1}, 0 \ldots, 0\right)}\left(u_{n}(x)+1\right)\right| .
$$

Lemma 4.1 With $u_{n}$ as above, there is $C>0$ and a positive integer $n_{1}$ so that for $n \geq n_{1}$

$$
\int_{D_{2 n}} \frac{1}{h^{\iota_{1}+\kappa_{1}}}\left|D^{\alpha-\left(\iota_{1}, 0, \ldots, 0\right)}\left(u_{n}(x)+1\right) D^{\beta-\left(\kappa_{1}, 0 \ldots, 0\right)}\left(u_{n}(x)+1\right)\right| d x \leq \frac{C}{h}
$$

for all $\alpha, \beta,|\alpha|=2 i-1,|\beta|=2 j-1, i, j \in\{1,2, \ldots, N\}, \iota_{1} \in\{1,2, \ldots, 2 i-1\}$, $\kappa_{1} \in\{1,2, \ldots, 2 j-1\}, \iota_{1}+\kappa_{1} \geq 1$.

Proof. In view of lemma 2.2, we know $u_{n}+1$ is close to 0 uniformly for $x \in D_{2 n}$. According to our assumption $W^{\prime \prime}(-1)>0$, we find

$$
\left(u_{n}(x)+1\right)^{2} \leq C W\left(u_{n}(x)\right)
$$

for $x \in D_{2 n}$ where $C$ is independent of large $n$. We then obtain

$$
\int_{D_{2 n}}\left(u_{n}(x)+1\right)^{2} d x \leq C
$$

where $C$ again is independent of large $n$. We also know that from the positivity of $\left[A_{\alpha \beta}\right]$

$$
\int_{\Omega}\left[D^{\alpha} u_{n}(x)\right]^{2} d x \leq C,|\alpha|=2 i-1, i=1,2, \ldots, N
$$


Appealing to (2.3) again, we deduce

$$
\int_{\Omega}\left[D^{\alpha} u_{n}(x)\right]^{2} d x \leq C,|\alpha|=2 i, i=1,2, \ldots, N-1 .
$$

Lemma 4.1 follows from these two bounds and Holder's inequality.

We sum over $\alpha, \beta$ and find

$$
\begin{gathered}
\int_{D_{2 n}} \sum_{i, j=1}^{N} \sum_{\substack{|\alpha|=2 i-1 \\
|\beta|=2 j-1}} A_{\alpha \beta} D^{\alpha} v_{n} D^{\beta} v_{n} d x \leq \int_{D_{2 n}} \xi^{2} \sum_{i, j=1}^{N} \sum_{\substack{\alpha|=2 i-1\\
| \beta \mid=2 j-1}} A_{\alpha \beta} D^{\alpha} u_{n} D^{\beta} u_{n} d x \\
+O\left(\frac{1}{h}\right) \leq \int_{D_{2 n}} \sum_{i, j=1}^{N} \sum_{\substack{|\alpha|=2 i-1 \\
|\beta|=2 j-1}} A_{\alpha \beta} D^{\alpha} u_{n} D^{\beta} u_{n} d x+O\left(\frac{1}{h}\right) .
\end{gathered}
$$

Together with (4.7) and the fact $v_{n} \in \mathcal{A}$, we deduce

$$
\begin{aligned}
& I \leq E\left(v_{n}\right)=\int_{\Omega}\left[\sum_{i, j=1}^{N} \sum_{\substack{|\alpha|=2 i-1 \\
|\beta|=2 j-1}} A_{\alpha \beta} D^{\alpha} v_{n} D^{\beta} v_{n}+W\left(v_{n}\right)\right] d x \\
& \leq \int_{M_{2 n}}\left[\sum_{i, j=1}^{N} \sum_{\substack{|\alpha|=2 i-1 \\
|\beta|=2 j-1}} A_{\alpha \beta} D^{\alpha} u_{n} D^{\beta} u_{n}+W\left(u_{n}\right)\right] d x+O\left(\frac{1}{h}\right) .
\end{aligned}
$$

If we choose $h$ large enough, we obtain

$$
\begin{gathered}
E\left(u_{n}\right) \geq \int_{\Omega \backslash M_{2 n}} W\left(u_{n}\right) d x+\int_{M_{2 n}}\left[\sum_{i, j=1}^{N} \sum_{\substack{\| \alpha|=2 i-1\\
| \beta \mid=2 j-1}} A_{\alpha \beta} D^{\alpha} u_{n} D^{\beta} u_{n}+W\left(u_{n}\right)\right] d x \\
\geq \lambda+I-3 \epsilon
\end{gathered}
$$

which implies that $\left\{u_{n}\right\}$ can not be an energy minimizing sequence.

Case 2: Suppose, taking a subsequence if necessary, the $u_{n}$ 's stay close to 1 in $D_{1 n}$.

This possibility can be ruled out by the same argument as in the previous case. Instead of considering $D_{2 n}$ and $M_{2 n}$, one considers $D_{1 n}$ and $M_{1 n}$. One takes

$$
v_{n}(x)=\left(u_{n}(x)-1\right) \xi\left(\frac{-x^{1}+\left(y_{n}^{1}-\left(r+r_{n}\right) / 2\right)}{h}\right)+1 .
$$

Case 3: Suppose, taking a subsequence if necessary, the $u_{n}$ 's stay close to -1 in $D_{1 n}$ and 1 in $D_{2 n}$. 
We truncate $u_{n}$ outside $B_{\Omega}\left(y_{n}, r_{n}\right)$ and replace it by $v_{n}$. These have approximately equal energies being at least close to $I$ there, but the two tails of $u_{n}$ have extra energies bounded away from 0 . Therefore such $u_{n}$ 's can not constitute an energy minimizing sequence. Set

$$
\begin{gathered}
w_{n}=\left\{\left[\left(u_{n}(x)+1\right) \xi\left(\frac{x^{1}-\left(y_{n}^{1}-\left(r+r_{n}\right) / 2\right)}{h}\right)-1\right]-1\right\} \\
\times \xi\left(\frac{-x^{1}+\left(y_{n}^{1}+\left(r+r_{n}\right) / 2\right)}{h}\right)+1
\end{gathered}
$$

where $h<\left(r_{n}-r\right) / 2$ is a large number to be specified later.

We first compare $\int_{B_{\Omega}\left(y_{n}, r_{n}\right)} W\left(u_{n}\right) d x$ with $\int_{\Omega} W\left(w_{n}\right) d x$. Observe that $u_{n}$ and $w_{n}$ only differ in $M_{1 n} \cup M_{2 n}$ for large $n$, and outside $B_{\Omega}\left(y_{n}, r_{n}\right), W\left(w_{n}\right)=0$. Also in $D_{1 n}$, where $u_{n}$ is close to $-1, w_{n}$ is closer to -1 , and in $D_{2 n}$, where $u_{n}$ is close to $1, w_{n}$ is closer to 1 . Therefore we deduce

$$
\int_{\Omega} W\left(w_{n}\right) d x \leq \int_{B_{\Omega}\left(y_{n}, r_{n}\right)} W\left(u_{n}\right) d x .
$$

We then compare

$$
\int_{B_{\Omega}\left(y_{n}, r_{n}\right)} A_{\alpha \beta} D^{\alpha} u_{n} D^{\beta} u_{n} d x \text { with } \int_{\Omega} A_{\alpha \beta} D^{\alpha} w_{n} D^{\beta} w_{n} d x .
$$

We consider the integrals on three different sets, $D_{1 n}, D_{2 n}$ and $B_{\Omega}\left(y_{n}, r\right)$. Since $u_{n}$ and $w_{n}$ agree in $B_{\Omega}\left(y_{n}, r\right)$ for large $n$. We only consider $D_{1 n}$ and $D_{2 n}$.

In $D_{1 n}$,

$$
\xi\left(\frac{x^{1}+\left(y_{n}^{1}+\left(r+r_{n}\right) / 2\right)}{h}\right)=1
$$

for large $n$. Therefore

$$
w_{n}(x)=\left[u_{n}(x)+1\right] \xi\left(\frac{x^{1}-\left(y_{n}^{1}-\left(r+r_{n}\right) / 2\right)}{d}\right)-1 .
$$

In $D_{2 n}$,

$$
\xi\left(\frac{x^{1}-\left(y_{n}^{1}-\left(r+r_{n}\right) / 2\right)}{h}\right)=1
$$

for large $n$. Therefore

$$
w_{n}(x)=\left[u_{n}(x)-1\right] \xi\left(\frac{-x^{1}+\left(y_{n}^{1}+\left(r+r_{n}\right) / 2\right)}{h}\right)+1 .
$$

Using the same argument as the one in case 1, we find

$$
\int_{\Omega} \sum_{i, j=1}^{N} \sum_{\substack{|\alpha|=2 i-1 \\|\beta|=2 j-1}} A_{\alpha \beta} D^{\alpha} w_{n} D^{\beta} w_{n} d x
$$




$$
\leq \int_{B_{\Omega}\left(y_{n}, r_{n}\right)} \sum_{i, j=1}^{N} \sum_{\substack{|\alpha|=2 i-1 \\|\beta|=2 j-1}} A_{\alpha \beta} D^{\alpha} u_{n} D^{\beta} u_{n} d x+O\left(\frac{1}{h}\right) .
$$

Together with (4.11) and the fact $w_{n} \in \mathcal{A}$ we deduce

$$
\begin{gathered}
I \leq \int_{\Omega}\left[\sum_{i, j=1}^{N} \sum_{\substack{|\alpha|=2 i-1 \\
|\beta|=2 j-1}} A_{\alpha \beta} D^{2 i-1} w_{n} D^{\alpha} w_{n}+W\left(w_{n}\right)\right] d x \\
\leq \int_{B_{\Omega}\left(y_{n}, r_{n}\right)}\left[\sum_{\substack{|\alpha|=2 i-1 \\
|\beta|=2 j-1}} A_{\alpha \beta} D^{\alpha} u_{n} D^{\beta} u_{n}+W\left(u_{n}\right)\right] d x+O\left(\frac{1}{h}\right) .
\end{gathered}
$$

If we choose $h$ large enough, we obtain

$$
\begin{gathered}
E\left(u_{n}\right) \geq \int_{\Omega \backslash B_{\Omega}\left(y_{n}, r_{n}\right)} W\left(u_{n}\right) d x \\
+\int_{B_{\Omega}\left(y_{n}, r_{n}\right)}\left[\sum_{i, j=1}^{N} \sum_{\substack{|\alpha|=2 i-1 \\
|\beta|=2 j-1}} A_{\alpha \beta} D^{\alpha} u_{n} D^{\beta} u_{n}+W\left(u_{n}\right)\right] d x \geq \Lambda-\lambda+I-3 \epsilon
\end{gathered}
$$

which implies that $\left\{u_{n}\right\}$ can not be an energy minimizing sequence if we choose $\epsilon$ small. This rules out the third possibility and proves lemma 3.3.

\section{A sufficient condition for non-periodic $\Omega$}

In this section we will study problem (1.1) in a non-periodic tube. The lack of periodicity causes the loss of translation. This brings a subtle difference between problem (1.1) in a periodic tube and that in a non-periodic tube. We will only consider non-periodic tubes that are "half-periodic" near the two ends. In this case, the concentration-compactness principle can give a sufficient condition which ensures the existence of a transition layer solution.

Let $\Omega$ be an open connected set in $R^{d}$. Suppose $\Omega$ can be divided into three mutually disjoint connected open sets $\Omega_{1}, \Omega_{2}$ and $\Omega_{0}$ where $\bar{\Omega}=\overline{\Omega_{1}} \cup \overline{\Omega_{2}} \cup \overline{\Omega_{0}}$. We further assume that $\Omega_{0}$ is bounded and $\Omega_{1}$ and $\Omega_{2}$ are "half periodic". That $\Omega_{1}$ is half periodic means there exists $T_{1} \in R^{d}$ such that for every $x_{1} \in \Omega_{1}$, $x_{1}+T_{1} \in \Omega_{1}$, and for every $x_{1} \in \Omega_{1}$ there exist $y_{1} \in \Omega_{0}$ and a non-negative integer $n_{1}$ satisfying $x_{1}=y_{1}+n_{1} T_{1}$. The same condition applies to $\Omega_{2} . \Omega_{1}$ and $\Omega_{2}$ are indeed the two half periodic tails of $\Omega$. We associate the end $E_{1}$ to $\Omega_{1}$ and the other end $E_{2}$ to $\Omega_{2}$ (see figure 2).

The function class $\mathcal{A}$ is defined in (2.5). For $u \in \mathcal{A}$ we require

$$
\lim _{x \rightarrow E_{1}} u(x)=-1, \lim _{x \rightarrow E_{2}} u(x)=1 .
$$



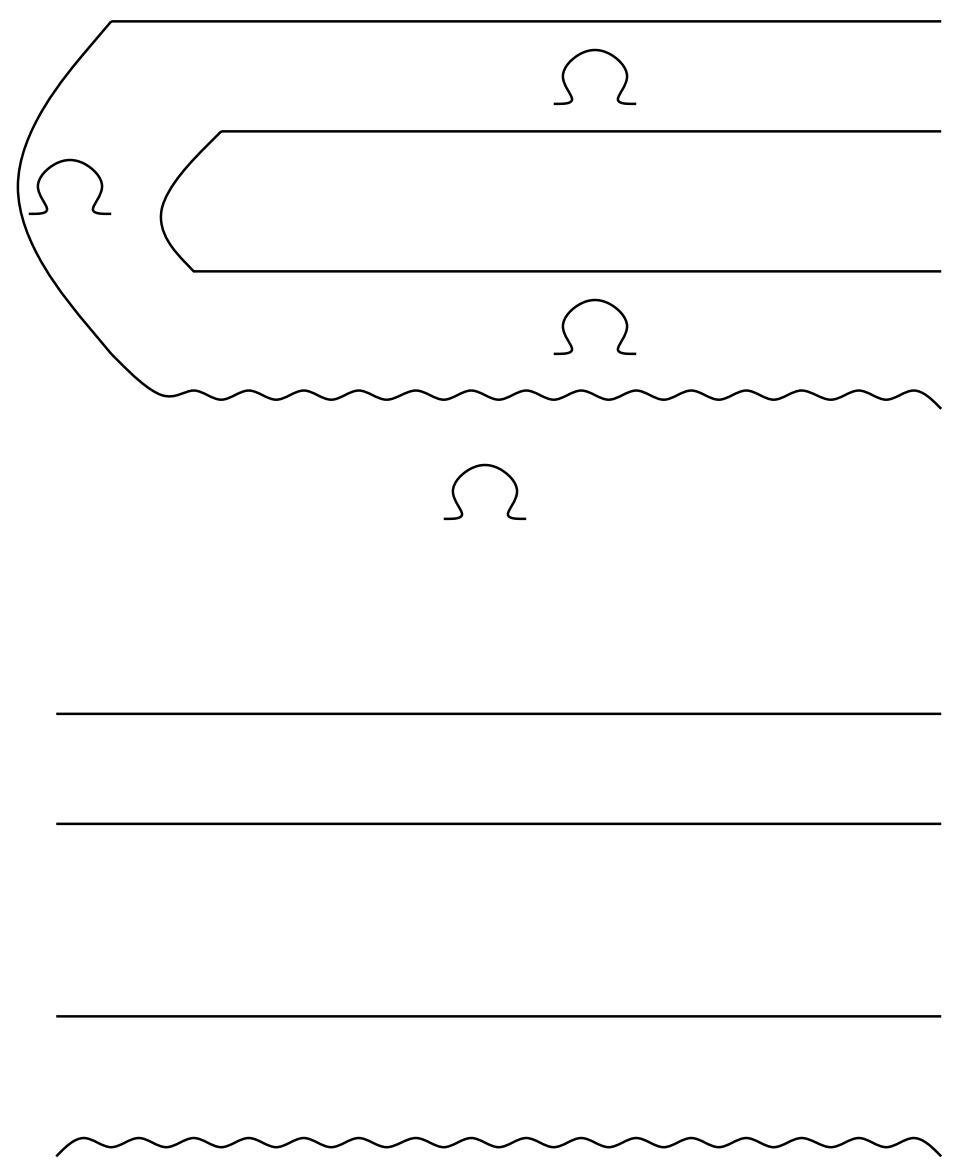

Figure 2: An example of non-periodic tubes. 
We define $G_{1}\left(G_{2}\right.$, respectively) to be the "periodic extension" of $\Omega_{1}\left(\Omega_{2}\right.$, respectively).

$$
G_{1}=\left\{x \in R^{d}: x+n_{1} T_{1} \in \Omega_{1} \text { for some integer } n_{1}\right\} .
$$

We require that $G_{1}\left(G_{2}\right.$, respectively) has bounded cross sections perpendicular to $T_{1}\left(T_{2}\right.$, respectively, where $T_{2}$ is the period of $G_{2}$.) We denote the other end of $G_{1}\left(G_{2}\right.$, respectively) by $E_{1}^{\prime}\left(E_{2}^{\prime}\right.$, respectively) (see figure 2$)$.

We now define two auxiliary variational problems:

$$
\begin{aligned}
& I_{1}=\inf _{\mathcal{A}_{1}} \int_{G_{1}}\left[\sum_{i, j=1}^{N} \sum_{\substack{|\alpha|=2 i-1 \\
|\beta|=2 j-1}} A_{\alpha \beta} D^{\alpha} u D^{\beta} u+W(u)\right] d x, \\
& I_{2}=\inf _{\mathcal{A}_{2}} \int_{G_{2}}\left[\sum_{i, j=1}^{N} \sum_{\substack{|\alpha|=2 i-1 \\
|\beta|=2 j-1}} A_{\alpha \beta} D^{\alpha} u D^{\beta} u+W(u)\right] d x .
\end{aligned}
$$

The function classes $\mathcal{A}_{1}$ and $\mathcal{A}_{2}$ are defined in a way similar to (2.5). We require for $u \in \mathcal{A}_{1}, \lim _{x \rightarrow E_{1}} u(x)=-1, \lim _{x \rightarrow E_{1}^{\prime}} u(x)=1$, and for $u \in \mathcal{A}_{2}$, $\lim _{x \rightarrow E_{2}^{\prime}} u(x)=-1, \lim _{x \rightarrow E_{2}} u(x)=1$.

Theorem 5.1 Let $\Omega$ be a tube half-periodic near the two ends. We always have

$$
I \leq \min \left\{I_{1}, I_{2}\right\}
$$

where $I$ is defined in (1.1) and $I_{1}, I_{2}$ are defined in (5.2). If the strict inequality

$$
I<\min \left\{I_{1}, I_{2}\right\}
$$

holds, then problem (1.1) has an energy minimizer in $\mathcal{A}$.

Proof. Without the loss of generality, we assume $I_{1} \leq I_{2}$. We know from theorem 3.1 that the infimum $I_{1}$ can be achieved by a function, say $v$, in $\mathcal{A}_{1}$. We assume without losing the generality $T_{1}=\left(T_{1}^{1}, 0 \ldots, 0\right), T_{1}^{1}<0$ and $(0,0, \ldots, 0) \in$ $\Omega_{1}$. Set

$$
v_{n}(x)=\left\{\begin{array}{l}
{\left[v\left(x+n T_{1}\right)-1\right] \xi\left(\frac{-x^{1}}{h}\right)+1, x \in \Omega_{1},} \\
1, x \in \Omega \backslash \Omega_{1}
\end{array}\right.
$$

where $\xi$ is defined in (4.5) and $n$ is a positive integer. Note $v_{n} \in \mathcal{A}$. We compare the energy of $v_{n}$ in $\mathcal{A}$ with that of $v$ in $\mathcal{A}_{1}$. When $n$ is large, $v_{n}$ is close to 1 for $x^{1} \in[-h, 0]$, so

$$
\int_{\Omega} W\left(v_{n}\right) d x=\int_{\substack{x \in \Omega \\ x_{1}<0}} W\left(v_{n}\right) \leq \int_{G_{1}} W(v) d x .
$$

Consider

$$
A_{\alpha \beta} D^{\alpha} v_{n} D^{\beta} v_{n}=
$$




$$
\begin{aligned}
& A_{\alpha \beta}\left[\sum_{\iota_{1} \leq \alpha_{1}} \frac{\alpha !}{\iota_{1} !} D^{\alpha-\left(\iota_{1}, 0, \ldots, 0\right)}\left(v\left(x+n T_{1}\right)-1\right) D^{\iota_{1}} \xi\left(\frac{-x^{1}}{h}\right)\right] \\
& \times\left[\sum_{\kappa_{1} \leq \beta_{1}} \frac{\beta !}{\kappa_{1} !} D^{\beta-\left(\kappa_{1}, 0, \ldots, 0\right)}\left(v\left(x+n T_{1}\right)-1\right) D^{\kappa_{1}} \xi\left(\frac{-x^{1}}{h}\right)\right] .
\end{aligned}
$$

The first term from the product is

$$
A_{\alpha \beta} D^{\alpha} v\left(x+n T_{1}\right) D^{\beta} v\left(x+n T_{1}\right) \xi^{2}\left(\frac{-x^{1}}{h}\right)
$$

and the other terms assume the form

$$
\begin{gathered}
\frac{A_{\alpha \beta} \alpha ! \beta !}{h^{\iota_{1}+\kappa_{1}} \iota \iota_{1} ! \kappa_{1} !} D^{\alpha-\left(\iota_{1}, \ldots, 0\right)} v\left(x+n T_{1}\right) D^{\beta-\left(\kappa_{1}, 0, \ldots, 0\right)} v\left(x+n T_{1}\right) \\
\times \xi^{\left(\iota_{1}\right)}\left(\frac{-x^{1}}{h}\right) \xi^{\left(\kappa_{1}\right)}\left(\frac{-x^{1}}{h}\right)
\end{gathered}
$$

where $\iota_{1}+\kappa_{1} \geq 1$. We sum over $\alpha, \beta$ and integrate over $\Omega$. We find, as in the proof of lemma 3.3,

$$
\begin{gathered}
\int_{\Omega} \sum_{i, j=1}^{N} \sum_{\substack{|\alpha|=2 i-1 \\
|\beta|=2 j-1}} A_{\alpha \beta} A_{\alpha \beta} D^{\alpha} v_{n} D^{\beta} v_{n} d x \\
=\int_{G_{1} \cap\left\{x^{1}>-1-n T_{1}^{1}\right\}} \sum_{\substack{i, j=1 \\
|\alpha|=2 i-1 \\
|\beta|=2 j-1}}^{N} A_{\alpha \beta} D^{\alpha} v D^{\beta} v d x+O\left(\frac{1}{h}\right) .
\end{gathered}
$$

Together with (5.4), we deduce

$$
\begin{gathered}
I \leq \int_{\Omega}\left[\sum_{i, j=1}^{N} \sum_{\substack{\alpha|=2 i-1\\
| \beta \mid=2 j-1}} A_{\alpha \beta} A_{\alpha \beta} D^{\alpha} v_{n} D^{\beta} v_{n}+W\left(v_{n}\right)\right] d x \\
\leq \int_{G_{1} \cap\left\{x^{1}>-1-n T_{1}^{1}\right\}} \sum_{i, j=1}^{N} \sum_{\substack{\alpha|=2 i-1\\
| \beta \mid=2 j-1}} A_{\alpha \beta} D^{\alpha} v D^{\beta} v d x+\int_{G_{1}} W(v) d x+O\left(\frac{1}{h}\right) .
\end{gathered}
$$

If we choose $h$ comparable with $n$ and send $n \rightarrow \infty$, we find

$$
I \leq \int_{G_{1}}\left[\sum_{i, j=1}^{N} \sum_{\substack{|\alpha|=2 i-1 \\|\beta|=2 j-1}} A_{\alpha \beta} D^{\alpha} v D^{\beta} v+W(v)\right] d x=I_{1} .
$$

This proves the first part of theorem 5.1.

We now assume $I<\min \left\{I_{1}, I_{2}\right\}$. We again consider the concentration functions, the $Q_{n}$ 's and $Q$, of the $W\left(u_{n}\right)$ 's where $\left\{u_{n}\right\}$ is an energy minimizing sequence in $\mathcal{A}$. We consider the three cases $\lambda=0, \lambda \in(0, \Lambda)$ and $\lambda=\Lambda$. As in 
the proof of theorem 3.1, we can rule out the cases $\lambda=0$ and $\lambda \in(0, \Lambda)$. We are left with the case $\lambda=\Lambda$ which implies from statement I in section 2 that there exist a subsequence of $u_{n}$, again denoted by $u_{n}$, and $y_{n} \in \Omega$ such that for every $\epsilon>0$ there exists $r>0$ so that

$$
\int_{B_{\Omega}\left(y_{n}, r\right)} W\left(u_{n}(x)\right) d x \geq \Lambda-\epsilon
$$

for large $n$.

We will prove that the $y_{n}$ 's indeed stay in a bounded subset of $\Omega$. Suppose the contrary, so we can assume a subsequence of $\left\{y_{n}\right\}$, still denoted by $\left\{y_{n}\right\}$ approaches $E_{1}$. Without the loss of generality, we also assume $(0,0, \ldots, 0) \in \Omega_{1}$, $T_{1}=\left(T_{1}^{1}, 0, \ldots, 0\right), T_{1}^{1}<0$. Define in $G_{1}$

$$
v_{n}(x)=\left\{\begin{array}{l}
\left(u_{n}(x)-1\right) \xi\left(\frac{-x^{1}}{h}\right)+1, x \in \Omega_{1} \\
1, x \in G_{1} \backslash \Omega_{1}
\end{array}\right.
$$

Here we first choose $h<-y_{n}^{1}-r$.

We observe since the $u_{n}$ 's are close to 1 in $\Omega_{0} \cup \Omega_{2}$,

$$
\int_{G_{1}} W\left(v_{n}\right) d x=\int_{\Omega_{1}} W\left(v_{n}\right) d x \leq \int_{\Omega} W\left(u_{n}\right) d x .
$$

As in the proof of lemma 3.3 , we write

$$
\begin{aligned}
A_{\alpha \beta} D^{\alpha} v_{n}(x) & D^{\beta} v_{n}(x)=A_{\alpha \beta} D^{\alpha}\left[\left(u_{n}(x)-1\right) \xi\left(\frac{-x^{1}}{h}\right)\right] D^{\beta}\left[\left(u_{n}(x)-1\right) \xi\left(\frac{-x^{1}}{h}\right)\right] \\
= & A_{\alpha \beta}\left[\sum_{\iota_{1} \leq \alpha_{1}} \frac{\alpha !}{\iota_{1} !} D^{\alpha-\left(\iota_{1}, 0 \ldots, 0\right)}\left(u_{n}(x)-1\right) D^{\iota_{1}} \xi\left(\frac{-x^{1}}{h}\right)\right] \\
& \times\left[\sum_{\kappa_{1} \leq \beta_{1}} \frac{\beta !}{\kappa_{1}} D^{\beta-\left(\kappa_{1}, 0, \ldots, 0\right)}\left(u_{n}(x)-1\right) D^{\kappa_{1}} \xi\left(\frac{-x^{1}}{h}\right)\right]
\end{aligned}
$$

where the first term from the product is

$$
A_{\alpha \beta} \xi^{2}\left(\frac{-x^{1}}{h}\right) D^{\alpha} u_{n} D^{\beta} u_{n}
$$

and the other terms assume the form

$$
\frac{A_{\alpha \beta} \alpha ! \beta !}{h^{\iota_{1}+\kappa_{1}} \iota_{1} ! \kappa_{1} !} D^{\alpha-\left(\iota_{1}, 0 \ldots, 0\right)}\left(u_{n}-1\right) D^{\beta-\left(\kappa_{1}, 0 \ldots, 0\right)}\left(u_{n}-1\right) \xi^{\left(\iota_{1}\right)}\left(\frac{-x^{1}}{h}\right) \xi^{\left(\kappa_{1}\right)}\left(\frac{-x^{1}}{h}\right),
$$

$\iota_{1}+\kappa_{1} \geq 1$. Summing over $\alpha, \beta$ and integrating over $G_{1}$, we find, as in the proof of lemma 3.3,

$$
\int_{G_{1}} \sum_{i, j=1}^{N} \sum_{\substack{|\alpha|=2 i-1 \\|\beta|=2 j-1}} A_{\alpha \beta} D^{\alpha} v_{n} D^{\beta} v_{n} d x=\int_{\Omega_{1}} \sum_{i, j=1}^{N} \sum_{\substack{|\alpha|=2 i-1 \\|\beta|=2 j-1}} A_{\alpha \beta} D^{\alpha} v_{n} D^{\beta} v_{n} d x
$$




$$
\begin{aligned}
& \leq \int_{\Omega_{1}} \sum_{i, j=1}^{N} \sum_{\substack{\alpha|=2 i-1\\
| \beta \mid=2 j-1}} A_{\alpha \beta} D^{\alpha} u_{n} D^{\beta} u_{n} d x+O\left(\frac{1}{h}\right) \\
& \leq \int_{\Omega} \sum_{i, j=1}^{N} \sum_{\substack{|\alpha|=2 i-1 \\
|\beta|=2 j-1}} A_{\alpha \beta} D^{\alpha} u_{n} D^{\beta} u_{n} d x+O\left(\frac{1}{h}\right) .
\end{aligned}
$$

Together with (5.5), we deduce

$$
\begin{gathered}
I_{1} \leq \int_{G_{1}}\left[\sum_{i, j=1}^{N} \sum_{\substack{|\alpha|=2 i-1 \\
|\beta|=2 j-1}} A_{\alpha \beta} D^{\alpha} v_{n} D^{\beta} v_{n}+W\left(v_{n}\right)\right] d x \\
\leq \int_{\Omega}\left[\sum_{i, j=1}^{N} \sum_{\substack{\alpha|=2 i-1\\
| \beta \mid=2 j-1}} A_{\alpha \beta} D^{\alpha} u_{n} D^{\beta} u_{n}+W\left(u_{n}\right)\right] d x+O\left(\frac{1}{h}\right)=I+O\left(\frac{1}{h}\right) .
\end{gathered}
$$

If we choose $h$ comparable with $-y_{n}^{1}$ and send $n \rightarrow \infty$, we obtain $I_{1} \leq I$ which contradicts the assumption $I<\min \left\{I_{1}, I_{2}\right\}$.

Once we know the $y_{n}$ 's stay in a bounded subset of $\Omega$, we conclude that there exists $u \in \mathcal{A}$ such that passing to a subsequence if necessary

$$
\begin{gathered}
u_{n} \rightarrow u \text { in } C(K) \text { for every compact } K \subset \bar{\Omega}, \\
D^{\alpha} u_{n} \rightarrow D^{\alpha} u \text { weakly in } L^{2}(\Omega),|\alpha|=2 i-1, i=1,2, \ldots, N .
\end{gathered}
$$

As in the proof of lemma 3.4, this $u$ is an energy minimizer of (1.1).

Similar to corollary 3.5 , we have

Corollary 5.2 If $I<\min \left\{I_{1}, I_{2}\right\}$, the convergence in (5.7) and (5.8) is indeed

$$
u_{n} \rightarrow u \text { in } C(\bar{\Omega}),
$$

$$
D^{\alpha} u_{n} \rightarrow D^{\alpha} u \text { strongly in } L^{2}(\Omega),|\alpha|=2 i-1, i=1,2, \ldots, N \text {. }
$$

\section{Some applications of theorem 5.1}

We will construct a few examples of $\Omega$ to illustrate theorem 5.1 in this section. We assume that equation (1.5) has the property of unique continuation, namely if a solution $u$ of (1.5) is equal to a constant in an open subset of $\Omega$, then $u$ is necessarily a constant in $\Omega$. If $W$ is real analytic, then the solutions of (1.5) are real analytic and (1.5) in turn has the property of unique continuation.

Our first example is a near periodic tube $\Omega_{A}$ in $R^{2}$. The corresponding periodic extensions $G_{1}, G_{2}$ of the tails are congruent to $G_{A}$ (see figure 3 ). Note that the cross sections of $\Omega_{A}$ have the same lengths as those of the corresponding cross sections of $G_{A}$ except in a middle region where the cross sections of $\Omega_{A}$ have shorter lengths than that of $G_{A}$. 


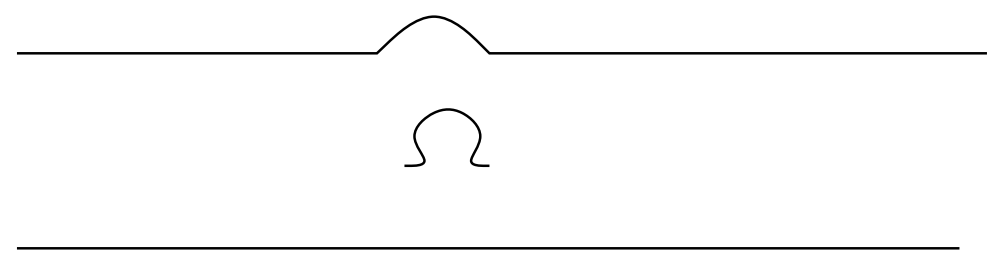

Figure 3: The picture of $\Omega_{A}$.

Let $u_{A}$ be an energy minimizer of problem (1.1) in $G_{A}$. The existence of such an energy minimizer is guaranteed by theorem 3.1. Denote this problem by

$$
I_{G}=\inf _{u \in \mathcal{A}_{G}} \int_{G_{A}}\left[\sum_{i, j=1}^{N} \sum_{\substack{\alpha|=2 i-1\\| \beta \mid=2 j-1}} A_{\alpha \beta} D^{\alpha} u D^{\beta} u+W(u)\right] d x .
$$

Clearly $I_{G}=I_{1}=I_{2}$ where $I_{1}$ and $I_{2}$ are the infima of the corresponding auxiliary problems. Take $u_{A}$ to be a test function and put it in $\Omega_{A}$. Because the middle region of $\Omega_{A}$ is thinner than that of $G_{A}$, we have

$$
\begin{gathered}
I \leq \int_{\Omega_{A}}\left[\sum_{i, j=1}^{N} \sum_{\substack{|\alpha|=2 i-1 \\
|\beta|=2 j-1}} A_{\alpha \beta} D^{\alpha} u_{A} D^{\beta} u_{A}+W\left(u_{A}\right)\right] d x \\
<\int_{G_{A}}\left[\sum_{i, j=1}^{N} \sum_{\substack{|\alpha|=2 i-1 \\
|\beta|=2 j-1}} A_{\alpha \beta} D^{\alpha} u_{A} D^{\beta} u_{A}+W\left(u_{A}\right)\right] d x=I_{G} .
\end{gathered}
$$

The strict inequality in (6.2) follows from the unique continuation which ensures that $u_{A}$ is not a constant in any open subset of $G_{A}$. Therefore in this tube there is an energy minimizer.

The second example is a near perfect tube except that somewhere in the middle there is a hole, but the lengths of the cross sections are identical. We denote the tube, its tails and their congruent periodic extension by $\Omega_{B}, \Omega_{B, 1}$, 

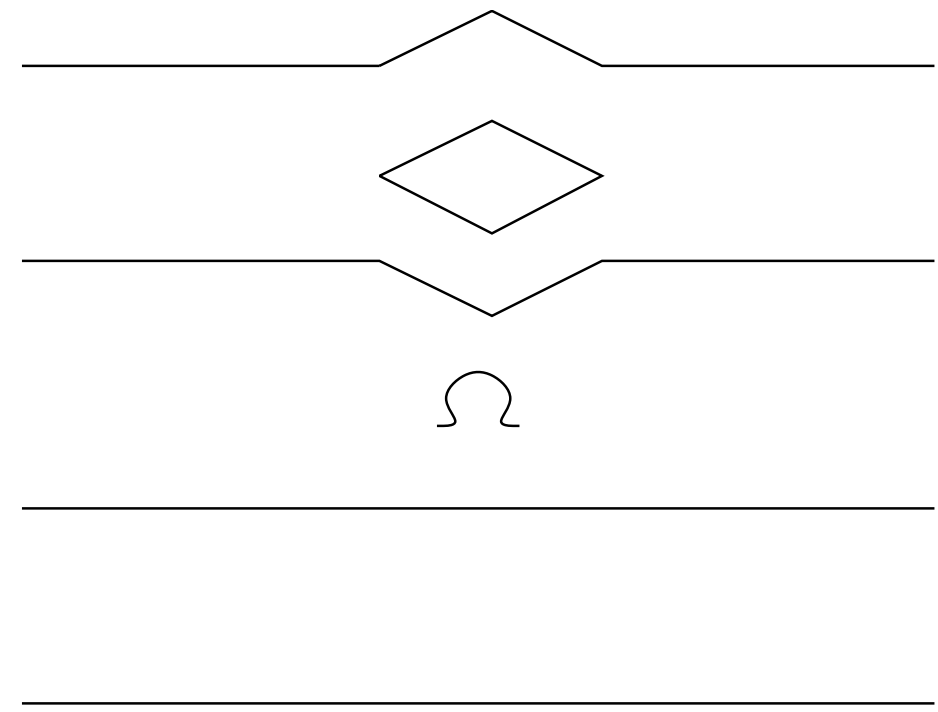

Figure 4: The picture of $\Omega_{B}$.

$\Omega_{B, 2}$ and $G_{B}$ (see figure 4 ). We take $u_{B}$ to be an energy minimizer of (1.1) in $G_{B}$. It is not clear to us whether $u_{B}$ is necessarily one-dimensional, i.e., $u_{B}$ is a function of $x_{1}$ only. If we are willing to make the assumption that $u_{B}$ is one-dimensional, then we extend $u_{B}$ to all $R^{2}$ in the obvious way and put it in $\Omega_{B}$. Because the cross sections of $\Omega$ have the same length, we have

$$
\begin{gathered}
\int_{\Omega_{B}}\left[\sum_{i, j=1}^{N} \sum_{\substack{|\alpha|=2 i-1 \\
|\beta|=2 j-1}} A_{\alpha \beta} D^{\alpha} u_{B} D^{\beta} u_{B}+W\left(u_{B}\right)\right] d x \\
=\int_{G_{B}}\left[\sum_{i, j=1}^{N} \sum_{\substack{|\alpha|=2 i-1 \\
|\beta|=2 j-1}} A_{\alpha \beta} D^{\alpha} u_{B} D^{\beta} u_{B}+W\left(u_{B}\right)\right] d x=I_{G} .
\end{gathered}
$$

However $u_{B}$ is not an energy minimizer in $\Omega_{B}$ since it does not satisfy the boundary conditions of the Euler-Lagrange equation (1.5) in $\Omega_{B}$. Therefore

$$
I<\int_{\Omega_{B}}\left[\sum_{i, j=1}^{N} \sum_{\substack{\alpha|=2 i-1\\| \beta \mid=2 j-1}} A_{\alpha \beta} D^{\alpha} u_{B} D^{\beta} u_{B}+W\left(u_{B}\right)\right] d x=I_{G}
$$

which again yields an energy minimizer in $\Omega_{B}$. 


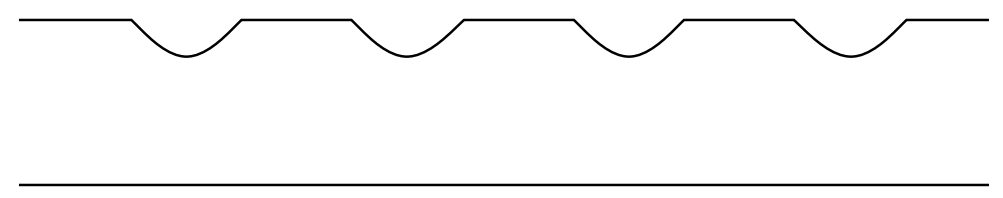

Figure 5: The picture of $\Omega_{C}$.

The third example will show that the sufficient condition is not always valid. Indeed in this example, problem (1.5) does not have an energy minimizer. Let $\Omega_{C}$ be a near perfect tube. Unlike the first example, we assume that somewhere in the tube the cross sections have longer lengths than that of the corresponding $G_{C}$ (see figure 5). If $u$ were an energy minimizer of (1.1) in $\Omega_{C}$, then we would have

$$
\begin{gathered}
I=\int_{\Omega_{C}}\left[\sum_{i, j=1}^{N} \sum_{\substack{|\alpha|=2 i-1 \\
|\beta|=2 j-1}} D^{\alpha} u D^{\beta} u+W(u)\right] d x \\
>\int_{G_{C}}\left[\sum_{i, j=1}^{N} \sum_{\substack{|\alpha|=2 i-1 \\
|\beta|=2 j-1}} D^{\alpha} u D^{\beta} u+W(u)\right] d x \geq I_{G}=I_{1}=I_{2} .
\end{gathered}
$$

This contradicts the first part of theorem 5.1.

Note added in proof. In a forthcoming paper, [11], the second named author is able to prove theorems 3.1 and 5.1 without condition (1.4).

\section{References}

[1] R.A. Adams, Sobolev Spaces, Academic Press, New York, 1975.

[2] P.W. Bates, P.C. Fife, R. Gardner and C.K.R.T. Jones, Phase field models for hypercooled solidification, preprint.

[3] P.W. Bates, P.C. Fife, R. Gardner and C.K.R.T. Jones, The existence of traveling wave solutions of a generalized phase-field model, preprint.

[4] P.W. Bates, P.C. Fife, X. Ren and X. Wang, Traveling waves in a convolution model for phase transitions, preprint.

[5] P.W. Bates and X. Ren, Heteroclinic orbits for a higher order phase transition problem, preprint.

[6] P. Fife and J.B. McLeod, The approach of solutions of nonlinear diffusion equations to traveling front solutions, Arch. Rat. Mech. Anal. 65 (1977), $335-361$. 
[7] R. Gardner and C.K.R.T. Jones, Traveling waves of a perturbed diffusion equation arising in a phase field model, Indiana Math. J. Vol 38, no. 4 (1989), 1197-1222.

[8] A.G. Khachaturyan, Microscopic theory of diffusion in crystalline solid solutions and the time evolution of the diffuse scattering of $\mathrm{X}$ rays and thermal neutrons, Soviet Phys. Solid State, Vol. 9 (1968), 2040-2046.

[9] A.G. Khachaturyan, Theory of Structural Transformations in Solids, Wiley-Interscience Publications, New York, 1983.

[10] P.L. Lions, The concentration-compactness principle in the calculus of variations: The locally compact case. Part I and Part II, Ann. Inset. H. Poincare Sect. A (N.S.) 1. (1984), 109-145, 223-283.

[11] X. Ren, Variational approach to multiple layers of the bistable equation in long tubes, preprint. 